\title{
Time-resolved optical absorption microspectroscopy of magnetic field sensitive flavin photochemistry
}

Lewis M. Antill, Joshua P. Beardmore, and Jonathan R. Woodward

Citation: Review of Scientific Instruments 89, 023707 (2018); doi: 10.1063/1.5011693

View online: https://doi.org/10.1063/1.5011693

View Table of Contents: http://aip.scitation.org/toc/rsi/89/2

Published by the American Institute of Physics

\section{Articles you may be interested in}

A novel multiplex absorption spectrometer for time-resolved studies

Review of Scientific Instruments 89, 024101 (2018); 10.1063/1.5006539

An efficient fluorescent single-particle position tracking system for long-term pulsed measurements of nitrogen-vacancy centers in diamond

Review of Scientific Instruments 89, 023702 (2018); 10.1063/1.5003707

Attosecond transient absorption instrumentation for thin film materials: Phase transitions, heat dissipation, signal stabilization, timing correction, and rapid sample rotation

Review of Scientific Instruments 89, 013109 (2018); 10.1063/1.4994041

Simultaneous AFM and fluorescence imaging: A method for aligning an AFM-tip with an excitation beam using a 2D galvanometer

Review of Scientific Instruments 89, 023708 (2018); 10.1063/1.5006724

A compact fluorescence polarization analyzer with high-transmittance liquid crystal layer Review of Scientific Instruments 89, 024103 (2018); 10.1063/1.5017081

A remotely triggered fast neutron detection instrument based on a plastic organic scintillator

Review of Scientific Instruments 89, 023115 (2018); 10.1063/1.5012121

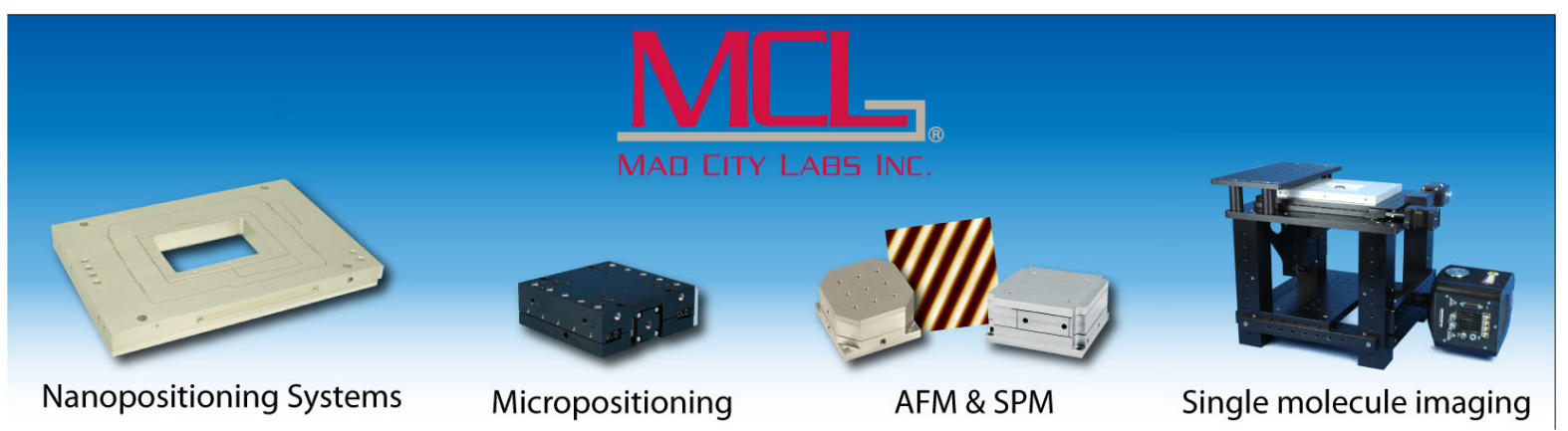




\title{
Time-resolved optical absorption microspectroscopy of magnetic field sensitive flavin photochemistry
}

\author{
Lewis M. Antill, ${ }^{1}$ Joshua P. Beardmore,$^{2}$ and Jonathan R. Woodward ${ }^{1}$ \\ ${ }^{1}$ Graduate School of Arts and Sciences, The University of Tokyo, 3-8-1 Komaba, Meguro-ku, Tokyo, Japan \\ ${ }^{2}$ Earth Dynamics Research Group, The Institute for Geoscience Research (TIGeR), \\ Department of Applied Geology, Bentley, Curtin University, Perth 6102, Australia
}

(Received 2 November 2017; accepted 3 February 2018; published online 28 February 2018)

\begin{abstract}
The photochemical reactions of blue-light receptor proteins have received much attention due to their very important biological functions. In addition, there is also growing evidence that the one particular class of such proteins, the cryptochromes, may be associated with not only a biological photo-response but also a magneto-response, which may be responsible for the mechanism by which many animals can respond to the weak geomagnetic field. Therefore, there is an important scientific question over whether it is possible to directly observe such photochemical processes, and indeed the effects of weak magnetic fields thereon, taking place both in purified protein samples in vitro and in actual biochemical cells and tissues. For the former samples, the key lies in being able to make sensitive spectroscopic measurements on very small volumes of samples at potentially low protein concentrations, while the latter requires, in addition, spatially resolved measurements on length scales smaller than typical cellular components, i.e., sub-micron resolution. In this work, we discuss a twoand three-color confocal pump-probe microscopic approach to this question which satisfies these requirements and is thus useful for experimental measurements in both cases. Published by AIP Publishing. https://doi.org/10.1063/1.5011693
\end{abstract}

\section{INTRODUCTION}

Recent experiments on magnetically sensitive animals including birds, ${ }^{1}$ fruit flies, ${ }^{2}$ and monarch butterflies ${ }^{3}$ have provided increasing amounts of circumstantial evidence that the class of blue-light receptor proteins called the cryptochromes may be involved in the process of detecting the geomagnetic field. It is proposed that spin correlated radical pairs (RPs) generated by photoinduced electron transfer from a protein residue (likely tryptophan) to a bound flavin adenine dinucleotide (FAD) cofactor molecule show magnetic field dependent kinetics via the radical pair mechanism (RPM) and that under the correct conditions, low field effects (LFEs) in this reaction might lead to the ability to detect magnetic fields as weak as that of the Earth $(\sim 30-50 \mu \mathrm{T}))^{4,5}$ Maeda and co-workers ${ }^{6}$ first demonstrated that cryptochrome purified from Arabidopsis thaliana exhibited magnetic field sensitive photochemistry when excited with nanosecond pulses of blue light in vitro, while a model radical pair system has demonstrated a measurable difference in photochemical kinetics in zero and Earth strength magnetic field. ${ }^{7}$

We describe in this paper, an instrument which aims to address two key experimental challenges in studying the magnetic field sensitivity of cryptochrome photochemistry:

1. Measurement sensitivity. The study of cryptochrome photochemistry requires the production of purified, cofactor bound samples of cryptochrome proteins from a variety of different species. For unravelling mechanistic aspects of the magnetoresponse, site-directed mutagenesis is extremely useful as it allows the modification of the structure in the region of the cofactor binding pocket and thus in the electron transfer, or other photochemical reaction processes, that can take place therein. Such experiments typically only produce small volumes (tens to thousands, in ideal cases, of microliters) of protein samples, typically at concentrations of tens or hundreds of micromoles per liter. This means that spectrometers capable of making sensitive time-resolved spectroscopic measurements on such samples are invaluable.

2. Spatially resolved measurements. Beyond the need to study the magnetic sensitivity of the photoreactions of isolated cryptochromes is the need to make corresponding measurements in cells and tissues, not least to address the question of anisotropic magnetic field responses, i.e., the ability to sense the direction of external magnetic fields.

The preliminary results obtained using our instrument were recently published elsewhere. ${ }^{8}$ In this paper, we provide details on our instrumental and experimental approach, demonstrating our technique's capabilities for use with biological samples and describe a methodology which allows the instrument to be used to make measurements on small volumes of samples in which the photochemical processes are non-cyclic, i.e., in which photoexcitation is destructive to the sample.

\section{EXPERIMENTAL}

\section{Reaction systems}

As a proxy for investigations on cryptochromes, we base our measurements on the photochemistry of the key cryptochrome cofactor, FAD, and observe photoinduced electron transfer reactions of this molecule in isolation, in which the 
intramolecular electron transfer can take place from the adenine moiety to the flavin moiety to generate a separated biradical at low $\mathrm{pH}$. Such a system represents the lower limit of sensitivity for observing the corresponding photochemistry in cryptochromes as the latter benefits from an optimal spatial arrangement of FAD and donor residues within the binding pocket. The efficiency of the electron transfer is substantially increased as evidenced by the fact that in the studies to date, RPs generated in cryptochrome (and the structurally similar photolyase protein from E. coli) are born in the singlet state, from the short-lived FAD excited singlet state, while FAD photochemistry in solution produces triplet born RPs, as the electron transfer is not fast enough to compete with intersystem crossing (ISC) in the excited FAD (Fig. 1). In the case of FAD alone at low $\mathrm{pH}$, there appears to be an equilibrium established between the protonated triplet state of FAD and the radical pair and so the total concentration of triplet state FAD and semiquinone radical combined are likely similar to the concentration of the protonated semiquinone radical produced in cryptochromes.

Our apparatus uses a blue laser to photoexcite the FAD molecule and then a (typically) green laser to probe the RP by detecting the one electron reduced semiquinone form of FAD produced by the electron transfer and the protonated triplet state of the flavin from which it is formed. Thus, the basic spectroscopic approach is the classic transient optical absorption spectroscopy, as used in the majority of studies of cryptochrome photochemistry to date.

For animals in the wild, blue-light irradiation conditions are quite different from the short (ns), intense laser pulses employed in flash photolysis measurements, being continuous wave and of considerably lower intensity. Therefore, it is important to be able to study the photochemistry under both sets of irradiation conditions. Our instrument allows measurements to be made in both irradiation regimes.
Indeed, it has been recently demonstrated ${ }^{9}$ that under appropriate reaction conditions, continuous blue-light irradiation of flavins can lead to chemical amplification of observed magnetic field effects (MFEs), which may be important in understanding the biological response.

\section{Instrumental details}

A schematic of the experimental arrangement is shown in Fig. 2. The pump and probe beams are provided by a pair of solid-state lasers [Coherent CUBE $449 \mathrm{~nm}$ (pump) and Coherent Sapphire $532 \mathrm{~nm}$ (probe)]. The output of the $532 \mathrm{~nm}$ laser is first beam split using a variable polarizing beam splitter before being combined with the output of the $449 \mathrm{~nm}$ laser using a dichroic mirror. The combined beams are then launched into a single mode optical fiber which conducts the laser light to the microscope apparatus. The single mode optical fiber ensures optimal spatial overlap and clean Gaussian beam profiles of the two laser beams used.

The microscope, shown in Fig. 3, is similar to an instrument used to image single molecules. ${ }^{10,11}$ It is constructed in a transmission confocal arrangement and utilizes the absorption (and/or scattering) of photons, at an appropriate wavelength, for imaging purposes.

The major components of the apparatus consist of a piezoelectric sample translation stage (PI nano ${ }^{\circledR}$ P-545), two super apochromat objective lenses (Olympus UPlanSApo20×), and a number of micrometer adjusted translation stages (Thorlabs). The use of the apochromatic lenses minimizes the relative displacement of the focal beam waists of the two wavelengths used to within $150 \mathrm{~nm}$, well within the Rayleigh lengths of the two beams. The beam waists are approximately $240 \mathrm{~nm}$ and $285 \mathrm{~nm}$ for the $449 \mathrm{~nm}$ and $532 \mathrm{~nm}$ beams, respectively. The microscope is assembled on a vibrationally isolated optical bench (Herz DT-8060M-D) to eliminate any potential external sources of spurious signals.
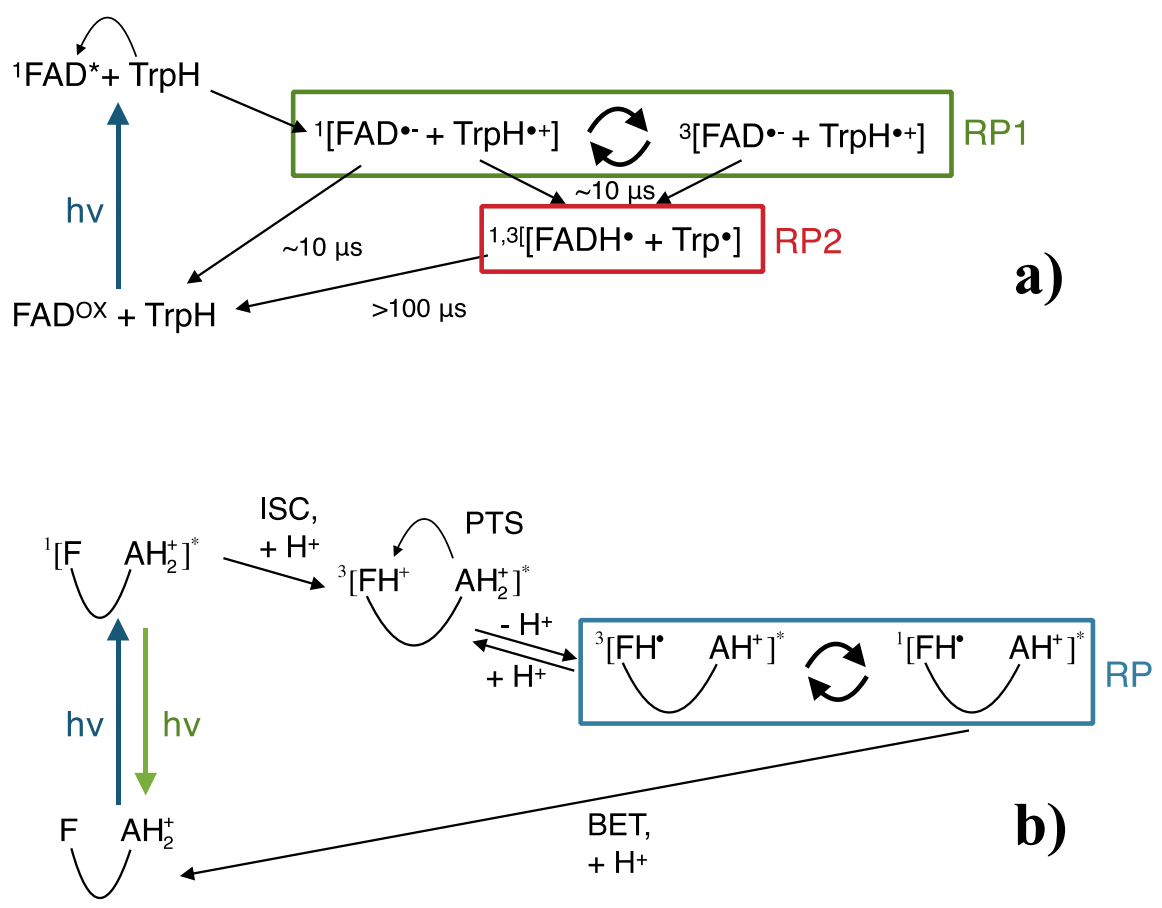

FIG. 1. Reaction scheme overviewing the spin selective photochemical reactions of FAD, (a) bound within $A$. thaliana cryptochromes (AtCry, based on Ref. 6) and (b) in acid solution. For AtCry, after photoexcitation, an electron is transferred along a chain of tryptophan (Trp) residues generating RP1 which undergoes spin selective back electron transfer (BET). RP1 is magnetically sensitive, and RP2 is long lived and magnetically insensitive. For FAD in acidic solution, (b), the electron transfer takes place to flavin $(F)$ from adenine (A) in the excited protonated triplet state [PTS, generated by intersystem crossing (ISC) from and protonation of the excited singlet state of protonated FAD] which generates a triplet born diradical which can undergo spin-selective BET. 


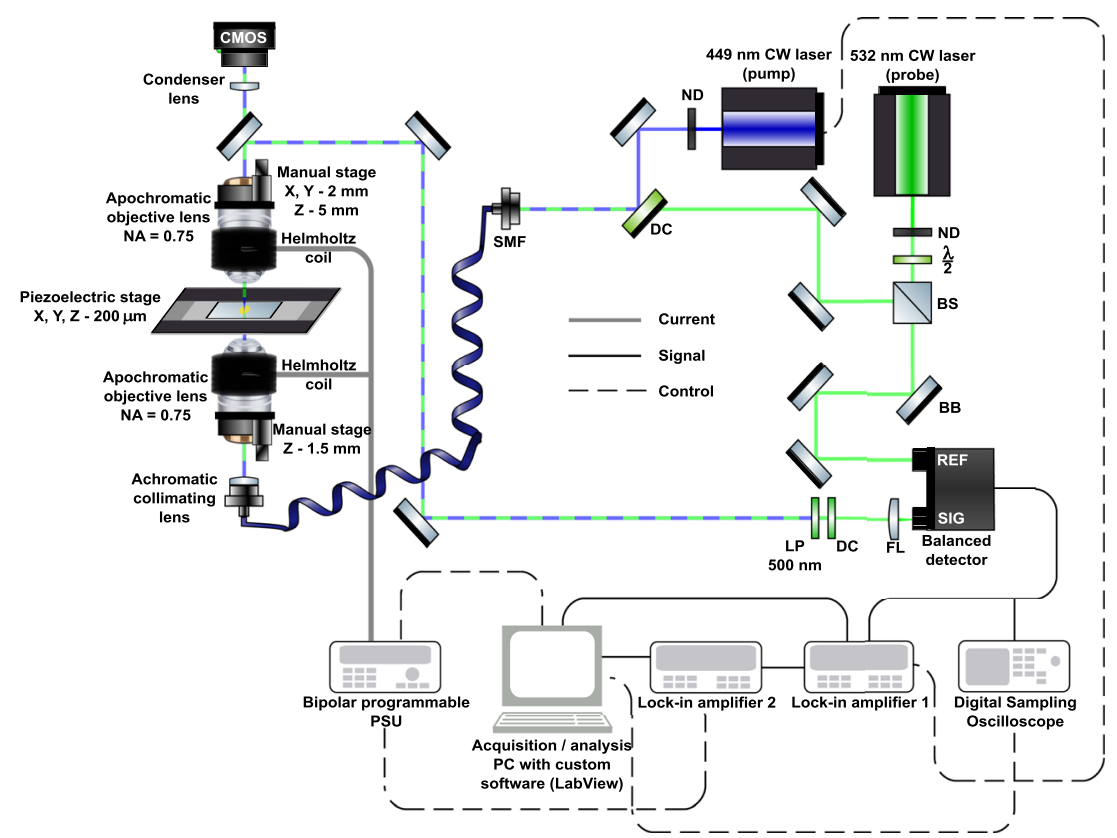

FIG. 2. Basic layout of the microscope. $\mathrm{SMF}=$ single mode fiber, $\mathrm{DC}=$ dichroic mirror, $\mathrm{ND}=$ neutral density filter, $\lambda / 2=$ half-wave plate polarizer, $\mathrm{BS}=$ beam splitter, $\mathrm{BB}=$ broadband dielectric mirror, $\mathrm{LP}=$ longpass filter, $\mathrm{FL}=$ focusing lens, $\mathrm{PSU}=$ power supply unit.
The optical axis of the microscope is defined by a $95 \mathrm{~mm}$ optical rail. This is mounted to an $\mathrm{x}$ - and $\mathrm{y}$-axis translation stage which enables $12.5 \mathrm{~mm}$ of travel across the sample. The microscope optics are mounted within a $30 \mathrm{~mm}$ cage system (Thorlabs) to allow accurate axial alignment and structural rigidity. The optical fiber from the laser system is introduced under the sample stage and is collimated at the optimal beam diameter for the objective lenses chosen. The lower objective lens is mounted on a $\mathrm{z}$-axis translation stage enabling the lens to sample distance to be adjusted with high precision. The upper objective can be adjusted along all three ( $\mathrm{x}-, \mathrm{y}-$, and $\mathrm{z}-$ ) axes and allows the pair of lenses to be accurately aligned into the confocal arrangement.

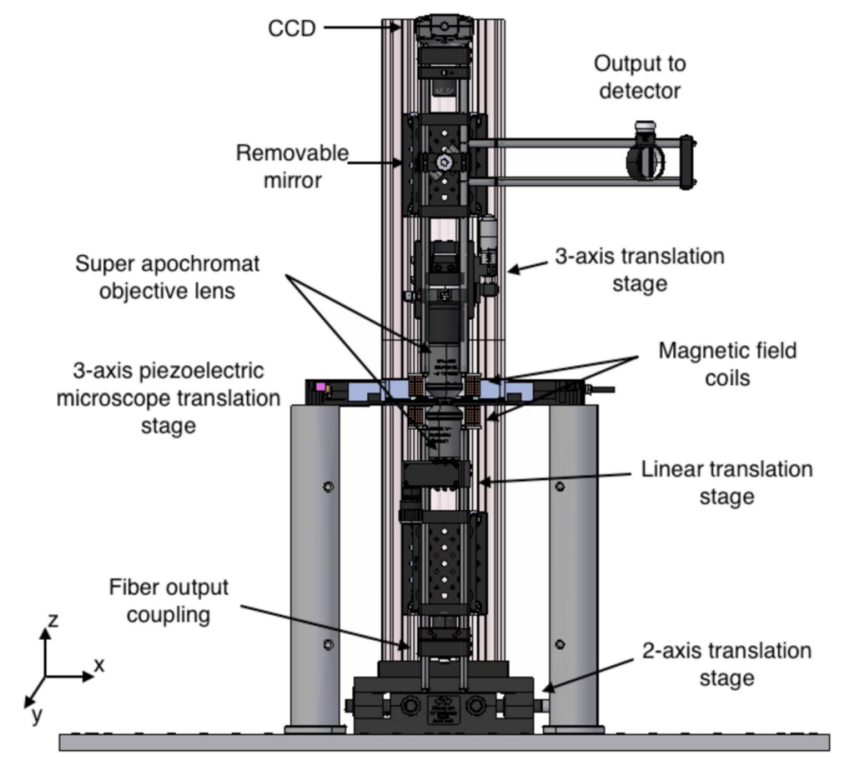

FIG. 3. Side-on-view of the main microscope unit, indicating the relative locations of the sample, objective lenses, magnetic field coils, and translation stages.
The output of the upper objective can be directed onto a CCD camera (Thorlabs DCC1645C-HQ), for alignment purposes, or reflected onto the input of either an auto-balanced photodetector (Nirvana, New Focus) or a fast-balanced photodetector when short transients are to be measured (Thorlabs PDB460A-AC). The reference for the balanced detection is provided by the split output of the $532 \mathrm{~nm}$ laser. Using this method, we are able to reduce the fluctuations in laser intensity by $>50 \mathrm{~dB}$. The output of the (auto-)balanced photodetector is then passed into a lock-in amplifier (LIA, Stanford Research Systems SR830 DSP) which enables the sensitive detection of small modulated signals in real time or alternatively to a digital sampling oscilloscope (DSO, Tektronix TDS7104 DPO) for direct observation of kinetic signals.

Magnetic fields are applied by a custom-built pair of coils, configured in the Helmholtz arrangement, and are powered using a computer controlled bi-polar power supply (Kikusui PBX 20-20). The coils are aligned to apply a magnetic field which is co-axial to the optical axis. The maximum DC magnetic field strength applied to the sample is approximately $45 \mathrm{mT}$ although for typical measurements a maximum field strength of $20 \mathrm{mT}$ is employed to ensure no problems with heating are encountered.

Initial alignment of the upper objective lens is performed whilst the lower objective is removed. This also allows regions of interest in the sample to be imaged using the CCD camera. Once centered on a region of interest, the lower objective is installed and the confocal arrangement is established using the $532 \mathrm{~nm}$ probe light. While this means that the arrangement is not truly confocal for the pump light, imaging and detection are only carried out using the probe light in this work. The arrangement is easily modified if detection of the pump light is to be performed. The output from the upper objective lens is then directed onto the detector through several long-pass filters (ensuring that no probe light reaches the detector) and enables the laser shot noise limited detection of the modulated absorption signal. In addition, if the probe beam is disabled, 
the experimental arrangement allows the direct confocal measurement of fluorescence from the sample, which can be useful for studying reaction systems in which the fluorescence allows monitoring of a spin-selective reaction.

\section{IMAGING METHODS}

We have already introduced new terms to describe the two different operational modes of the microscope: transient optical absorption detection (TOAD) and magnetic intensity modulation (MIM) imaging. ${ }^{8}$ We provide details on the instrumental aspects of these techniques below.

\section{TOAD (transient optical absorption detection)}

The basic principle behind the TOAD measurement is to measure the change induced in the probe light reaching the detector by modulation of the pump light. There are primarily two approaches-one for measuring the effect of a short pump pulse (Fig. 4(a)) and one for measuring the effect of a slower square wave modulation of the pump light (Fig. 4(b)). The latter allows the equilibrium state of a cyclic photochemical reaction to be reached and MFEs thereon to be measured, while the former is a classic flash-photolysis approach, measuring the decay of transient species generated in the laser flash. Slow pump-light modulation utilizes a transistortransistor logic (TTL) output of the lock-in amplifier to switch the output from the $449 \mathrm{~nm}$ laser. The laser is capable of being modulated at frequencies up to $150 \mathrm{MHz}$, though in this case is limited to around $102 \mathrm{kHz}$ due to the bandwidth of the detector. For measuring equilibrium reaction kinetics in flavin reactions, actual modulation frequencies employed are typically $1 \mathrm{kHz}$ or below. For flash photolysis measurements, the laser can also be modulated using a pulse generator (Stanford Research Systems DG535), which allows the pulse length to be adjusted down to about $300 \mathrm{~ns}$, limited by the laser diode driver circuitry. All data presented here were measured with this arrangement; however, in recent experiments, we have been able to reduce the pulse width to a minimum of around $50 \mathrm{~ns}$ by using a short pulse laser driver (PicoLAS LDP-V 10-70). This enables experiments to be performed under conditions similar to those used in conventional flash
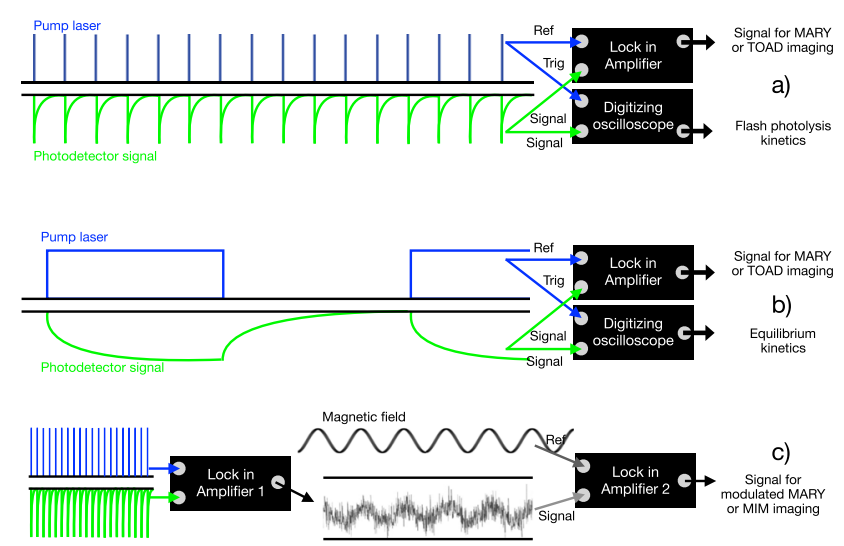

FIG. 4. Graphical representations summarizing the imaging methodologies. (a) TOAD imaging approach for the flash photolysis mode and (b) for slower square wave modulation. (c) The methodology for MIM imaging and modulated MARY. photolysis apparatus, allowing time-resolved optical absorption kinetic curves to be recorded from the photodetector. Using blue laser diodes with a $\mathrm{CW}$ output of up to $6 \mathrm{~W}$, effective flash photolysis measurements can be made by focusing the beam to the diffraction limit, despite the relatively low single pulse energy $(0.6 \mu \mathrm{J})$. In actual experiments, much lower laser powers are used. For cyclic flavin photoreactions, repetition rates of up to $10 \mathrm{kHz}$ provide rapid data acquisition while still providing sufficient sampling time to record complete kinetic transients and for the full reaction cycle to complete. In cryptochrome samples, slower repetition rates are likely to be necessary, a simple adjustment, due to the fact that our pump beam modulation is achieved by simple switching of a $\mathrm{CW}$ laser, meaning that modulation frequencies of $<1 \mathrm{~Hz}$ are readily obtained.

In the TOAD configuration, there are a number of different measurements that can be made. By fixing the position of the sample, direct flash photolysis or slow modulation kinetic traces can be captured by the DSO and such kinetics can be observed in the presence or absence of a magnetic field of selectable intensity. For the short pulse experiments, use of a fast photodetector allows direct measurement of the time dependence of the probe light following pump light excitation, giving direct kinetic information about the radical decay processes.

Both slow modulation and short pulse experiments can exploit lock-in detection at the modulation/pulse repetition frequency to provide a signal directly related to the amount of probe light absorbance, which is of great utility in imaging. To record a TOAD image, the focused beams are rastered across the region of the sample of interest using the $x$ - and $y$-axes of the piezo-stage which allows single steps down to $1 \mathrm{~nm}$ in all three dimensions. The output of the lock-in amplifier is converted to a relative absorbance and plotted against the $\mathrm{x}$ - and $y$-coordinates to generate the image. This means that a TOAD image effectively plots the signal that is proportional to the concentration of the probe beam absorbing transient species (in these measurements, the signal comes from both the FAD triplet state and the semiquinone radical, which are in rapid equilibrium). Slices through different layers of the sample can be obtained by controlling the $\mathrm{z}$-axis of the stage.

Under either modulation scheme, by stepping a DC magnetic field from negative to positive magnetic field strengths and by recording the lock-in detected signal at each field point, a curve indicating the magnetically affected reaction yield (MARY) can be quickly obtained. It is also possible to record the averaged kinetic trace at a given field position using the DSO and thus obtain a two-dimensional data set that comprises the variation of the absorption signal with time and magnetic field. Data extracted from this set can be used to plot the time dependence of the MARY spectrum.

\section{MIM (magnetic intensity modulation)}

As an alternative to modulating the pump light, it is possible to use constant pump light irradiation and instead modulate the applied magnetic field (magnetic intensity modulationMIM). The magnetic field is still stepped through multiple DC magnetic field strengths but also has a small AC magnetic field 
component. This is achieved using a sine-wave output of the lock-in amplifier and an external input into the bi-polar power supply. The amplitude and frequency of this input can be used to alter the sensitivity, and signal strength, of this technique. Larger modulation depths provide stronger signals but at the expense of removing sharp features, for example, the LFE. Very large magnetic field modulations can affect the stability of the microscope, and as such modulations in this study are limited to less than $1 \mathrm{mT}_{\mathrm{pk}-\mathrm{pk}}$. The magnetic field is modulated at frequencies $\leq 5 \mathrm{kHz}$.

This method results in data which are the first derivative (with respect to magnetic field strength) of those obtained using the TOAD method. This is a classic magnetic field modulation MARY experiment.

In practice, a much more effective approach, and the one actually used for all MIM measurements, is a combination of both previous techniques in a double modulation measurement (Fig. 4(c)). A second lock-in amplifier (NF Electronic Instruments LI-570A) is required for this measurement. The pump laser is pulsed at $>1 \mathrm{kHz}$ (typically $10 \mathrm{kHz}$ ) using short (typically $300 \mathrm{~ns}$ ) pulses from the delay generator. The phase sensitive detected absorption signal is then output directly from the first lock-in amplifier into the second. The second amplifier's internal signal generator is used to modulate the magnetic field, typically at around $100 \mathrm{~Hz}$ (which allows the use of appropriate time constants based on the averaging cycle of the first lock-in), and allows the detection of the component of the signal from the first lock-in amplifier that varies at this frequency. This AC magnetic field is combined with a static DC field, which can be varied to obtain the first derivative MARY spectrum, as mentioned previously, or which can be tuned to the value which provides maximum signal to use for magnetic sensitive imaging. Monitoring the output of the second lockin amplifier as the laser beams are scanned over the sample allows the direct recording of a magnetic intensity modulation (MIM) image. In such an image, the signal is only non-zero when there is a non-zero modulated absorption signal due to the effect of the pump laser, and there is a subsequent lower frequency modulation of that signal by the oscillating magnetic field. This approach results in substantially improved signal-to-noise ratios over that of the single field modulation approach. The main reason is that the primary optical signal is much more sensitive to vibrations and other fluctuations at frequencies in the range of hertz to hundreds of hertz. The first high frequency light modulation then acts as a filter to remove such fluctuations before using the lower frequency range for field modulation. This approach can, in principle, be used to image regions of biological cells and tissues to search for regions in which magnetically sensitive photochemistry is occurring. It is expected that experiments, which are currently underway, on the imaging of cultured cells and retinal tissue may allow identification of cells and cell components where such processes are occurring.

\section{MATERIALS}

The samples used in this work consist of flavin adenine dinucleotide (FAD) in a disodium phosphate/citric acid buffer solution, which allows the $\mathrm{pH}$ to be controlled over a wide range ( $\mathrm{pH}$ 2.2- $\mathrm{pH}$ 8.0). In some experiments, tryptophan was added as an external electron donor. All chemicals were used as supplied (Sigma-Aldrich). The concentrations used in each experiment are detailed in the text.

The solution is placed between two standard cover glasses, and the sample thickness can be set using an appropriate spacer. In the investigations discussed here, the sample thickness is set either using $100 \mu \mathrm{m}$ glass beads (Sigma-Aldrich) or $2.5 \mu \mathrm{m}$ polymer particles (Spherotech).

\section{RESULTS AND DISCUSSION}

In our previous paper, ${ }^{8}$ we demonstrated the imaging capability and spatial resolution of the microscope. Here we focus on the kinetic and MARY data that can be readily obtained from very small samples, even at low concentrations. Figure 5 shows a typical set of data that can be obtained from the standard TOAD configuration, for a $200 \mu \mathrm{M}$ sample of FAD in $\mathrm{pH} 2.3$ disodium phosphate/citric acid buffer in the presence of $1.5 \mathrm{mM}$ tryptophan. The sample was a $5 \mu \mathrm{l}$ spot of solution sandwiched between two glass cover slides at a separation of $100 \mu \mathrm{m}$ (achieved using glass spheres of this diameter as spacers). The operating repetition rate of photoexcitation is $10 \mathrm{kHz}$ which, in principle, allows a decay curve obtained from averaging 10000 shots to be recorded each second [in practice, acquisition is slower due to the retriggering rate of the DPO (Tektronix TDS7104 DPO)]. The kinetic curve in Fig. 5(a) is the result of averaging 30 such decay curves. Figure 5(b) shows the MARY curve obtained under the same conditions using lock-in detection with a $300 \mathrm{~ms}$ time constant and sweeping the magnetic field from positive to negative. The curve is an average of 4 individual MARY curves, each with an acquisition time of approximately $1 \mathrm{~min}$.

Studies to date have only examined the magnetic field effect on FAD at $\mathrm{pH}$ values less than about 3.6. ${ }^{12}$ It is believed
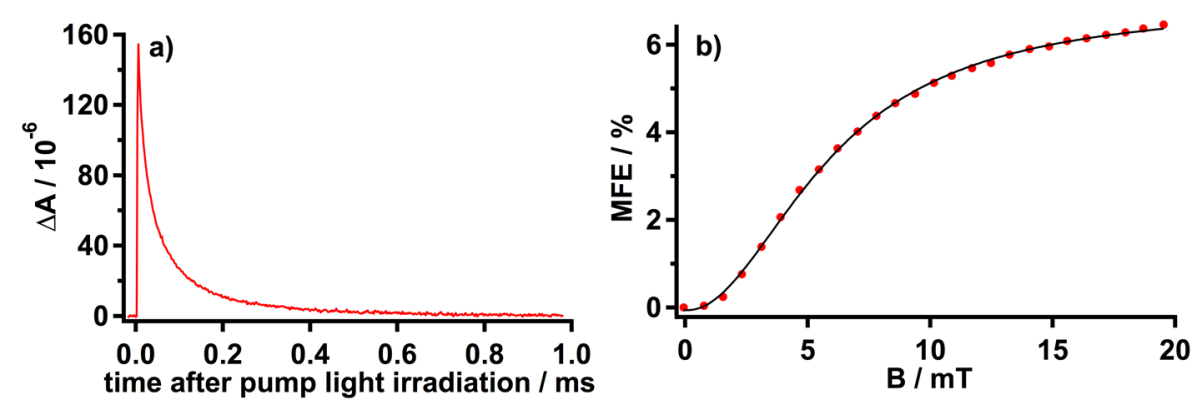

FIG. 5. (a) Time dependence of the $532 \mathrm{~nm}$ absorption signal $(\Delta \mathrm{A})$; (b) MARY spectrum for FAD $(200 \mu \mathrm{M})$ and $\operatorname{Trp}(1.5 \mathrm{mM})$ at $\mathrm{pH} 2.3$; the $\mathrm{B}_{1 / 2}$ value is $6.0 \pm 0.7 \mathrm{mT}$. 
that at higher $\mathrm{pH}$ the adenine moiety is not protonated and that FAD molecules adopt a closed configuration, in which the rapid (forward and backward) electron transfer between flavin and adenine moieties results in substantially diminished fluorescence quantum yield and halts RP formation. Due to the high sensitivity of our microspectroscope, we were able to study the reaction kinetics and field dependence at $\mathrm{pH}$ values up to $\mathrm{pH} 8.0$ and resolve not only the time-resolved optical absorption signals but also effects of a magnetic field thereon. In addition, by measuring the MARY curves at each $\mathrm{pH}$ value, we were able to measure the $\mathrm{pH}$ dependence of the $\mathrm{B}_{1 / 2}$ value (magnetic field strength at half the saturation value of the MFE, commonly used to characterize MFEs arising due to the hyperfine mechanism) for FAD (200 $\mu \mathrm{M})$ (Fig. 6) (a full analysis will appear in a future publication). These measurements demonstrate clearly that at physiological $\mathrm{pH}$ and higher, FAD is actually capable of producing radical pairs that are magnetic field sensitive. In addition, the drop in both the size of the absorption signal and the magnitude of the MFE demonstrates the sensitivity of the instrument and its potential utility for MFE measurements in cellular and tissue samples.

Finally, to demonstrate that our instrument can resolve very small field induced changes, rather than relatively large changes on weak absorption signals, we made measurements of riboflavin (RF) in water [Fig. 7(a)]. The primary photochemistry of RF in water is not field sensitive, as there is no appropriate electron donor to quench the photoexcited RF triplet state. However, it has been demonstrated that the reaction of two excited RF triplets can produce a pair of RF radical ions, ${ }^{13}$

$$
{ }^{3} \mathrm{RF}^{*}+{ }^{3} \mathrm{RF}^{*} \rightarrow \mathrm{RF}^{+}+\mathrm{RF}^{-},
$$

where

$$
\mathrm{RF}^{-} \rightarrow \mathrm{RFH} \cdot
$$

which forms a radical pair with another semiquinone radical, ${ }^{14}$

$$
\mathrm{RFH} \cdot+\mathrm{RFH} \cdot \rightarrow \mathrm{RFH}_{2}+\mathrm{RF} .
$$

The time-resolved optical absorption measurement on this signal is dominated by the RF photoexcited triplet state, but a very small magnetic field effect (of about $0.2 \%$ ) is observed and can be readily resolved as a clear MARY curve by our instrument [Fig. 7(b)].

The use of our instrument as a microspectroscope for MFE studies can be potentially extended to a wide-range of RP generating photochemical reactions. The primary limitation is that most photochemical processes that generate magnetically sensitive RPs are non-cyclic, i.e., the photochemical precursor is not regenerated in the reaction. Therefore, in instruments employed to date to study such reactions, the sample is usually refreshed using a flow system. To address this restriction and allow our instrument to make measurements on non-cyclic photoreactions, we have developed a sample rastering approach. For example, to record a MARY spectrum or
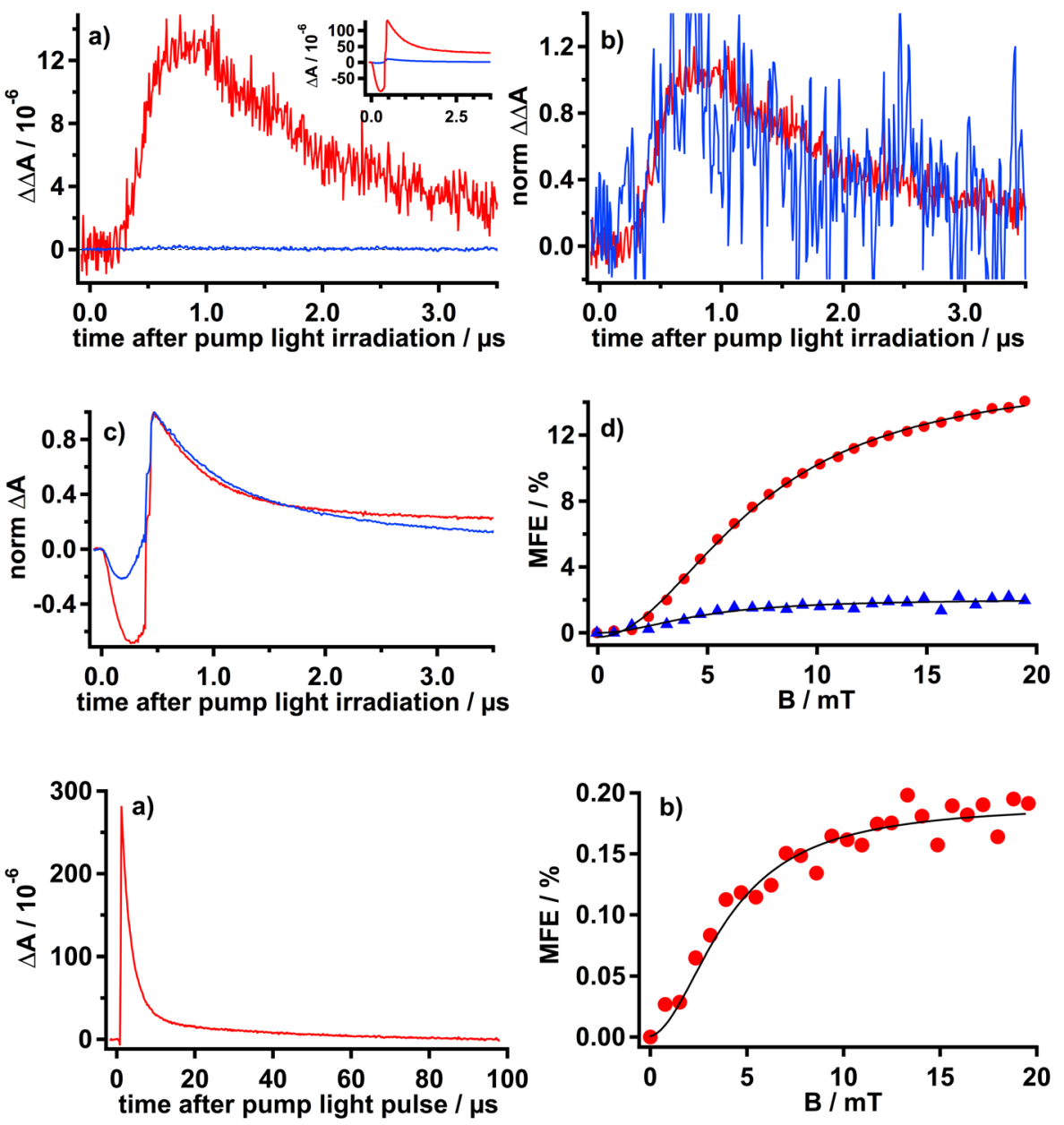

FIG. 6. pH dependence of FAD (200 $\mu \mathrm{M})(\mathrm{pH} 2.3=$ red and $\mathrm{pH} 8.0=$ blue $)$. (a) $\Delta \Delta \mathrm{A}[\Delta \Delta \mathrm{A}=\Delta \mathrm{A}(\mathrm{B}=20 \mathrm{mT})-$ $\Delta \mathrm{A}(\mathrm{B}=0 \mathrm{mT})]$ of $\sim 12 \times 10^{-6}$ and $\sim 2 \times 10^{-7}$ for $\mathrm{pH} 2.3$ and $\mathrm{pH} 8.0$, respectively, and inset $\triangle \mathrm{A}$ ( $\mathrm{x}$-axis has the same time scale as main), (b) normalized $\Delta \Delta \mathrm{A}$ $[\Delta \Delta \mathrm{A}=\Delta \mathrm{A}(\mathrm{B}=20 \mathrm{mT})-\Delta \mathrm{A}(\mathrm{B}=$ $0 \mathrm{mT}$ )], (c) normalized $\Delta \mathrm{A}$, (d) MARY curve $\mathrm{pH} 2.3$ (red dots) and $\mathrm{pH} 8.0$ (blue triangles); the $\mathrm{B}_{1 / 2}$ values are $7.3 \pm$ $1.0 \mathrm{mT}$ and $4.6 \pm 2.4 \mathrm{mT}$, respectively.
FIG. 7. Riboflavin in water. (a) Time dependence of the $532 \mathrm{~nm}$ absorption signal and (b) MARY spectrum for a pair of riboflavin radical ions in water; the $B_{1 / 2}$ value is $4.1 \pm 1.8 \mathrm{mT}$. 


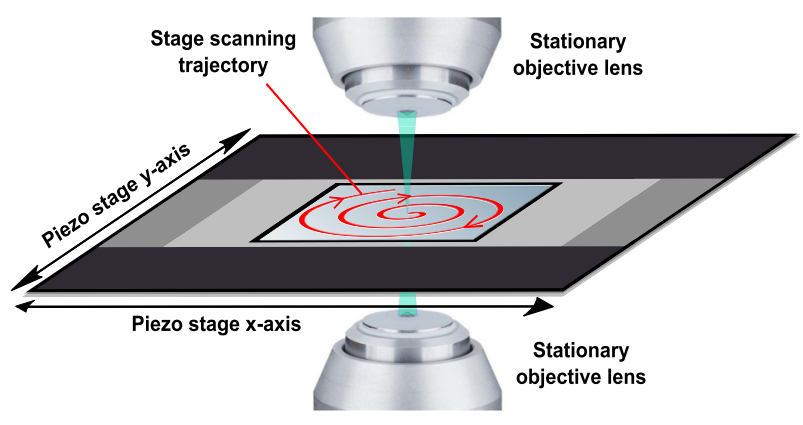

FIG. 8. Schematic of the spiral stage scanning principle.

a kinetic curve, after acquisition begins, the sample is moved using the piezoelectric stage such that a fresh sample is continuously introduced. To optimize this process, the piezo-stage operates in a manner that allows the sample to move outwards from the center in a spiral at a definable rate such that the sample is continuously replenished and the maximum sampling can be achieved for the minimum area of sample scanned (Fig. 8).

To demonstrate the utility of this technique, along with an additional advantage of being able to do sample replenishment for solid-state samples, we measured the MARY curve for a sample of riboflavin dissolved in a solid starch film. It has been previously shown that flavins embedded in a starch matrix can undergo a photochemical reaction involving glucose repeat units of the starch film (Ref. 15 and the references therein). The photoexcited fully oxidized riboflavin quinone undergoes intersystem crossing (ISC), electron transfer (ET), and proton transfer (PT) to produce a triplet born riboflavin semiquinone and glucose $\mathrm{C} 2 \mathrm{O}$ radical pair [Fig. 9(b)]. Surprisingly there is no evidence of exchange coupling in the MARY spectrum, which would be anticipated for a fixed RP with a small separation between the pair members. The simplest explanation is that both flavin molecules and semiquinone flavin radicals are mobile within the porous starch matrix such that radical pairs can undergo diffusive recombination, and therefore features due to level crossing are not observed. Upon further photoexcitation, subsequent ET and PT produce a fully reduced riboflavin hydroquinone and the photochemically degraded starch repeat unit, oxystarch. In the dark state, partial thermal re-oxidation (with starch restructuring) of the hydroquinone to the quinone groundstate is possible by the oxidizing action of the di-aldehyde groups of oxystarch; this process takes $\sim 4$ months [Fig. 9(a)]. Due to the long ground-state and starch repeat unit recovery time, our spiral scanning algorithm was able to replenish the sample, producing a MARY spectrum for the photochemical reaction between riboflavin and glucose repeat unit [Fig. 9(c)].

\section{Three-color approach—two probes and one pump}

The photochemistry of different flavin and tryptophan species has specific absorption wavelengths, which are discernible in transient absorption spectroscopic techniques. It is therefore valuable to utilize multiple wavelengths in a spectroscopic analysis, to develop a more concrete understanding of the photochemistry taking place in the chosen sample. This led us to develop a two-probe technique specific to the proposed magnetosensitive photochemistry of cryptochromes. ${ }^{6,16}$ The technique comprises three laser beams (each separated by $1.5 \mathrm{~mm}$ ), $449 \mathrm{~nm}$ (pump), $532 \mathrm{~nm}$ (probe), and $635 \mathrm{~nm}$ (probe, Thorlabs CPS635S), directly entering the objective lenses without fiber-coupling and with no beam expansion for back aperture filling. The pump beam passes through the center of the objective lenses, with a probe beam $1.5 \mathrm{~mm}$ on either side of the pump beam [Fig. 10(a)]. This technique allows us to record simultaneous MARY spectra and kinetic information at $532 \mathrm{~nm}$ and $635 \mathrm{~nm}$ using two (auto-) balanced detectors (Fig. 10) or alternatively by selectively steering the output and reference beams of either wavelength into the detector. This scheme can be extended to multiple different probe wavelengths due to the wide range of diode laser wavelengths available in the visible region. In our instrument, the primary limitation of two probe beam colors comes from the amount of available space on our optical bench.

a)

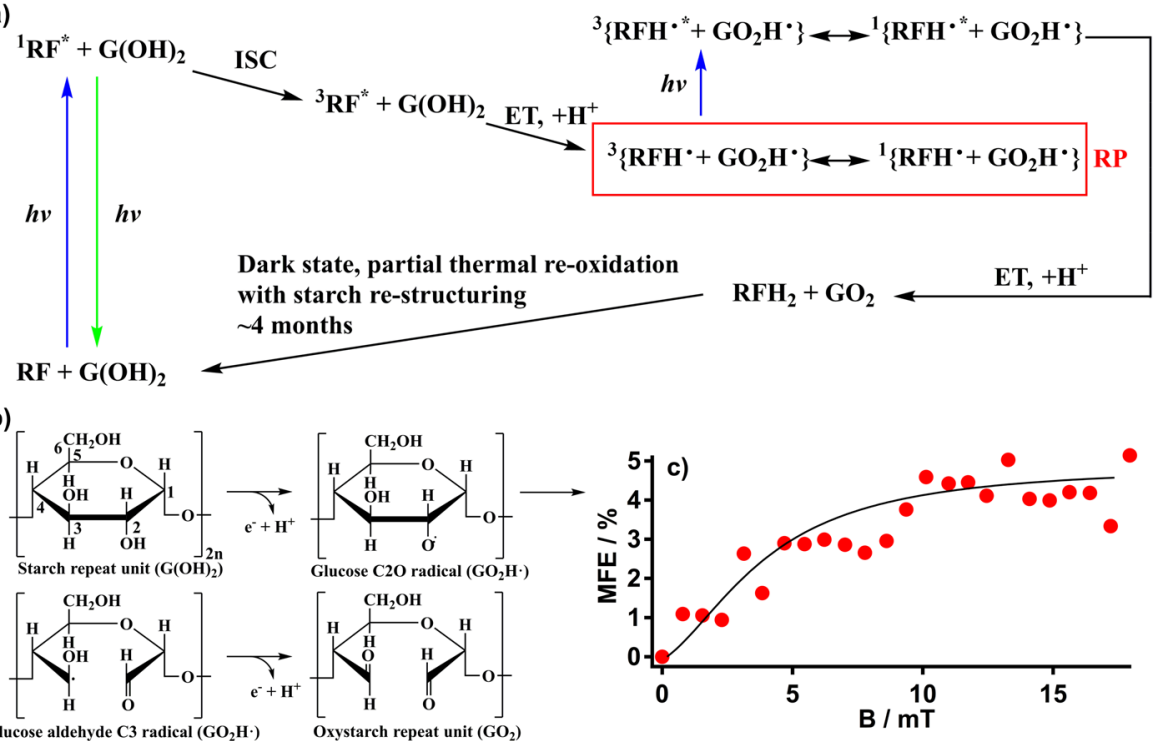

FIG. 9. (a) Photochemical reaction scheme for riboflavin and the glucose repeat unit of the starch thin film, (b) oxidation scheme of the glucose repeat unit of starch to the di-aldehyde glucose repeat unit of oxystarch (based on Ref. 15), (c) MARY spectrum displaying the magnetic field effect on the photochemical reaction between the riboflavin semiquinone and the oxidized glucose radicals; the $\mathrm{B}_{1 / 2}$ value is $4.6 \pm 2.7 \mathrm{mT}$. 


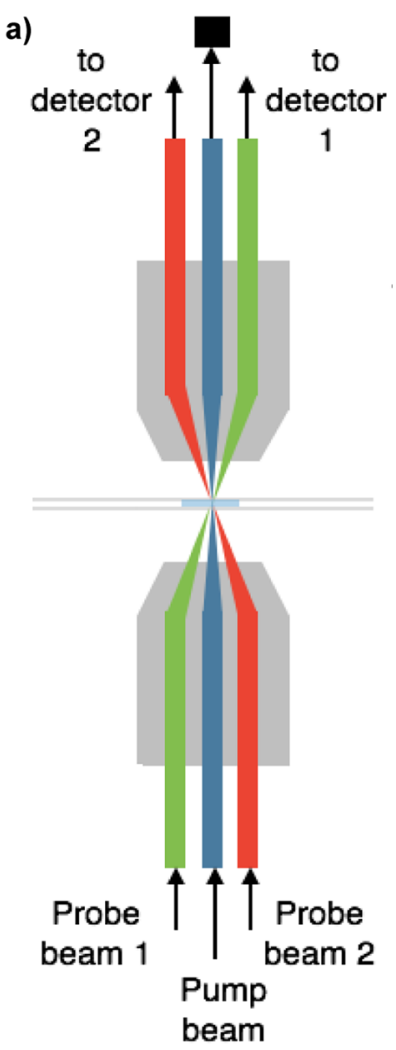

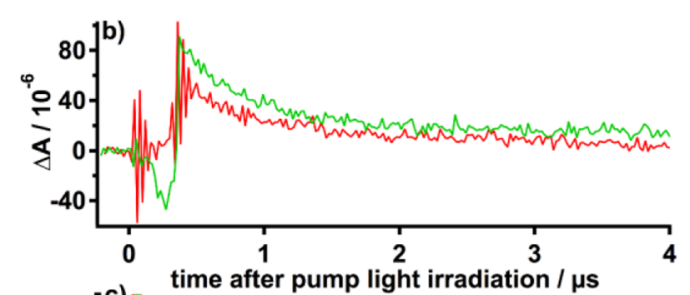
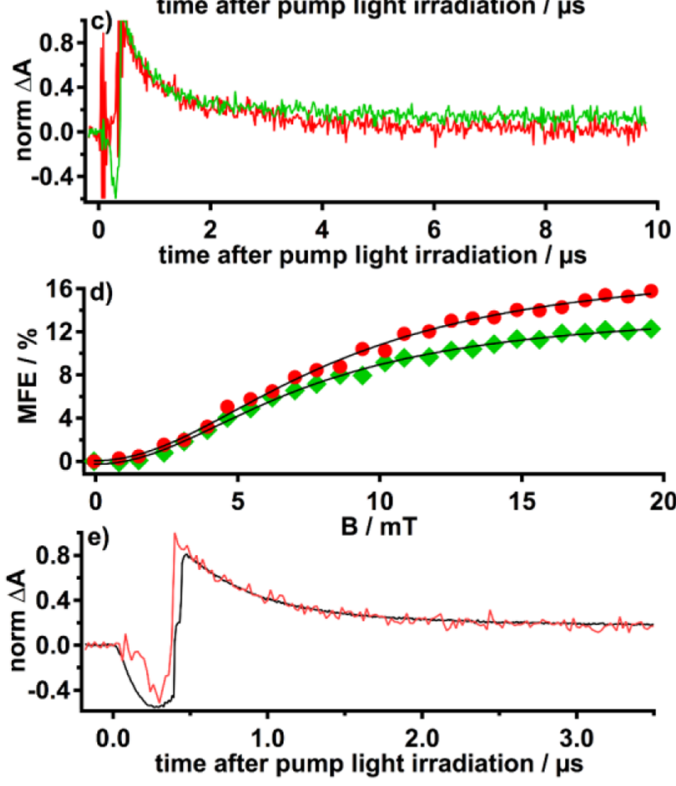

FIG. 10. Three-color approach. (a) Schematic of the separated beams experimental design. [(b)-(e)] Wavelength dependence on the kinetics and MFE for FAD $(200 \mu \mathrm{M})$ at $\mathrm{pH} 2.3$ [532 nm (green curve) and $635 \mathrm{~nm}$ (red curve)]. (b) $\Delta \mathrm{A}$ for $532 \mathrm{~nm}$ and $635 \mathrm{~nm}$, (c) normalized $\triangle \mathrm{A}$ for $532 \mathrm{~nm}$ and $635 \mathrm{~nm}$, (d) MARY curve at $635 \mathrm{~nm}$ (red dots) and $532 \mathrm{~nm}$ (green diamonds), $\mathrm{B}_{1 / 2}$ values are $8.4 \pm 2.0 \mathrm{mT}$ and $7.5 \pm$ $1.6 \mathrm{mT}$, respectively, (e) normalized $\Delta \mathrm{A}$ at $532 \mathrm{~nm}$ for fiber-coupled probe beam (black curve) and separated non-fiber-coupled probe beam (red curve).
The kinetic data for FAD (200 $\mu \mathrm{M}, \mathrm{pH} 2.3)$ in Fig. 10 illustrate that the $532 \mathrm{~nm}$ decay curve exhibits the presence of a long-lived species that is not observed in the $635 \mathrm{~nm}$ decay curve. This long-lived species is assigned to an intermediate generated from a bimolecular reaction at acidic $\mathrm{pH},{ }^{12,17}$ which also increases in life-time with increasing laser power. At $635 \mathrm{~nm}$, only a short-lived signal is observed, attributed to signals from both the RP (minor) and T-T absorption of the cation ${ }^{3} \mathrm{FH}^{+}$(major). ${ }^{12,18}$ The MARY spectra for the two wavelengths display a difference of $\sim 3 \%$. The lower MFE of $\sim 12 \%$ at $532 \mathrm{~nm}$ can be attributed to the long-lived species [which is not magnetic field (MF) sensitive] observed at this wavelength, as the \%MFE is calculated using the absolute detector signal from the LIA as an external MF is swept from -20 to $+20 \mathrm{mT}$. The $532 \mathrm{~nm}$ MARY spectra and kinetic curves are comparable for the separated and fiber-coupled beam experiments. An MFE of $\sim 12 \%$ for both experiments is observed, and the decay curves are identical [Fig. 10(e)] except for the early time fluorescence component, which is substantially reduced in the separated beam arrangement. This is due to the use of irises in the paths of the separated probe beams which means that the majority of the fluorescence signal does not reach the detector-unlike in the original arrangement. Alternatively, the arrangement can be used to capture the absorption signal at one wavelength using one detector and only fluorescence using the other. These two features are both substantial advantages of this arrangement. Essentially no fluorescence is observed for the $635 \mathrm{~nm}$ decay curve due to the weaker fluorescence signal at this wavelength. The signal-to-noise ratio is much poorer in the separated beam arrangement for conventional samples, where photoreaction takes place at all sample depths, but the vertical spatial resolution is increased as the beams only overlap in the beam waist region. In the combined beams arrangement, there is beam overlap across the entire width of the sample, leading to a substantially stronger signal. This may be an advantage for purely spectroscopic measurements while the separated beam arrangement should show comparable signal-to-noise ratios in true sub-micron scale measurements but with an inherent resolution reduction in all three dimensions due to the (possibly) non-Gaussian beams and lack of back aperture filling. In future work, the pump laser will be replaced by the new $6 \mathrm{~W}$ diode laser with a rapid current switching circuit, which will limit the pulse width to $50 \mathrm{~ns}$ or less and allow better discrimination of the absorption kinetics.

\section{SUMMARY AND CONCLUSIONS}

In this paper, we described the confocal magnetic field based microspectroscopic techniques of TOAD and MIM and demonstrated the excellent sensitivity of our instrumental arrangement by observing, for the first time, MFEs on the photochemistry of FAD at around biological $\mathrm{pH}(\Delta \Delta \mathrm{A} \sim 2 \times$ $10^{-7}$ ) and on the photochemistry of riboflavin (vitamin B2) in water $\left(\Delta \Delta \mathrm{A} \sim 6 \times 10^{-7}\right)$. The ability to work with non-cyclic reactions, using a spiral scanning approach, was confirmed through the observation of a MFE on the non-cyclic reaction between riboflavin and a thin film starch matrix at neutral $\mathrm{pH}$. The possibility of recording two probe wavelengths simultaneously was demonstrated in acidic FAD solution, and this arrangement is freely extendable to a much larger number of wavelengths, where sources, detectors, and bench space allow. The ability to make magnetic field measurements on photochemistry both in a spatially resolved manner and with 
high sensitivity in small volume, low concentration samples makes this a powerful tool in the armory of a spin chemist. The instrument is already proving useful in measuring flavin photochemistry in micron scale reactors, and measurements are underway to try to observe flavin photochemistry within living cells.

\section{ACKNOWLEDGMENTS}

L.M.A. and J.P.B. contributed equally to this work. The work was supported by JSPS KAKENHI Grant Nos. 24350002 and $15 \mathrm{~K} 13618$. The authors are grateful for a MEXT scholarship for L.M.A.

${ }^{1}$ W. Wiltschko, R. Wiltschko, and T. Ritz, Procedia Chem. 3, 276 (2011).

${ }^{2}$ R. J. Gegear, A. Casselman, S. Waddell, and S. M. Reppert, Nature 454, 1014 (2008).

${ }^{3}$ S. M. Reppert, P. A. Guerra, and C. Merlin, Annu. Rev. Entomol. 61, 25 (2016).

${ }^{4}$ T. Ritz, S. Adem, and K. Schulten, Biophys. J. 78, 707 (2000).

${ }^{5}$ P. J. Hore and H. Mouritsen, Annu. Rev. Biophys. 45, 299 (2016).
${ }^{6}$ K. Maeda, A. J. Robinson, K. B. Henbest, H. J. Hogben, T. Biskup, M. Ahmad, E. Schleicher, S. Weber, C. R. Timmel, and P. J. Hore, Proc. Natl. Acad. Sci. U. S. A. 109, 4774 (2012).

${ }^{7}$ K. Maeda, K. B. Henbest, F. Cintolesi, I. Kuprov, C. T. Rodgers, P. A. Liddell, D. Gust, C. R. Timmel, and P. J. Hore, Nature 453, 387 (2008).

${ }^{8}$ J. P. Beardmore, L. M. Antill, and J. R. Woodward, Angew. Chem., Int. Ed. 54, 8494 (2015).

${ }^{9}$ D. R. Kattnig, E. W. Evans, V. Déjean, C. A. Dodson, M. I. Wallace, S. R. Mackenzie, C. R. Timmel, and P. J. Hore, Nat. Chem. 8, 384 (2016).

${ }^{10}$ P. Kukura, M. Celebrano, A. Renn, and V. Sandoghdar, J. Phys. Chem. Lett. 1, 3323 (2010).

${ }^{11}$ M. Celebrano, P. Kukura, A. Renn, and V. Sandoghdar, Nat. Photonics 5, 95 (2011)

${ }^{12}$ M. Murakami, K. Maeda, and T. Arai, J. Phys. Chem. A 109, 5793 (2005).

${ }^{13}$ T. B. Melø, M. A. Ionescu, G. W. Haggquist, and K. Razi Naqvi, Spectrochim. Acta, Part A 55, 2299 (1999).

${ }^{14}$ V. Massey and G. Palmer, Biochemistry 5, 3181 (1966).

${ }^{15}$ A. Penzkofer, Int. J. Mol. Sci. 13, 9157 (2012).

${ }^{16}$ D. M. W. Sheppard, J. Li, K. B. Henbest, S. R. T. Neil, K. Maeda, J. Storey, E. Schleicher, T. Biskup, R. Rodriguez, S. Weber, P. J. Hore, C. R. Timmel, and S. R. Mackenzie, Sci. Rep. 7, 42228 (2017).

${ }^{17}$ M. S. Grodowski, B. Veyret, and K. Weiss, Photochem. Photobiol. 26, 341 (1977).

${ }^{18}$ M. Sakai and H. Takahashi, J. Mol. Struct. 379, 9 (1996). 